\title{
CAMBIOS EN PROPIEDADES QUÍMICAS Y BIOQUÍMICAS DEL SUELO ASOCIADOS AL REEMPLAZO DE PASTIZALES NATURALES POR PINUS RADIATA D. DON Y ROTACIONES AGRÍCOLAS
}

\section{CHANGES IN SOIL CHEMICAL AND BIOCHEMICAL PROPERTIES ASSOCIATED WITH REPLACEMENT OF NATURAL GRASSLANDS BY PINUS RADIATA D. DON AND AGRICULTURAL ROTATIONS}

\author{
Paula Florencia Di Gerónimo ${ }^{1,2 *}$, Cecilia Videla ${ }^{2}$, María Elena Fernandez ${ }^{3}$, Ester Cristina Zamuner², \\ y Pablo Laclau ${ }^{4}$ \\ ${ }^{1}$ Comisión de Investigaciones Científicas de la provincia de Buenos Aires (CIC), Calle 526 e/ 10 y 11 , \\ La Plata, Buenos Aires, Argentina \\ ${ }^{2}$ Facultad de Ciencias Agrarias, Universidad Nacional de Mar del Plata (FCA-UNMdP), Ruta 226 km \\ 74,5, Balcarce, Buenos Aires, Argentina \\ ${ }^{3}$ Consejo Nacional de Investigaciones Científicas y Técnicas (CONICET). Godoy Cruz 2290. Ciudad \\ Autónoma de Buenos Aires, Argentina. \\ ${ }^{4}$ Instituto Nacional de Tecnología Agropecuaria (INTA Bariloche), Modesta Victoria 4450, San Carlos \\ de Bariloche, Río Negro, Argentina. \\ *Autor para correspondencia E-mail: digeronimopaula@gmail.com
}

\section{RESUMEN}

El reemplazo de vegetación nativa de pastizales por agricultura, o por forestación, genera cambios en las propiedades del suelo afectando su productividad. El objetivo de este trabajo fue estudiar los cambios en algunas propiedades edáficas generados por los siguientes cambios en el uso de la tierra: 1) Pastizal Natural (PN) a agricultura (AGR); 2) PN a forestación con pinos (MP); y 3) Franjas raleadas dentro de MP: pastizal secundario (PS). Se tomaron muestras de suelo representativas de tales situaciones y se analizaron propiedades químicas y bioquímicas. Todos los sistemas produjeron una reducción del $\mathrm{pH}$ y aumento en la saturación con $\mathrm{H}^{+}$del suelo. En los sitios MP y PS se produjo un aumento del carbono orgánico total del suelo (COT). El $\mathrm{N}$ potencialmente mineralizable por incubación anaeróbica (Nan) de estos sitios fue muy inferior al de $\mathrm{PN}$, probablemente por diferencias en la calidad de su materia orgánica. PS disminuyó disminución en la acidez del suelo, y aumento del Nan y del P- extractable. En AGR se halló acidificación pero en menor grado, reducción del COT, Nan y P extractable. Los cambios observados indican que en sitios donde se reemplazó vegetación nativa por forestación con pinos, al retirarse la vegetación introducida, el suelo tiende rápidamente a recuperar sus características originales.

Palabras clave: Acidez, carbono del suelo, sistemas silvopastoriles.

\section{ABSTRACT}

The replacement of native vegetation of grasslands by agriculture, or by afforestation, causes changes in the properties of the soil, affecting its productivity. The objective of this research was to study the changes in some edaphic properties generated by the following changes in land use: 1) Natural grassland (PN) to agriculture (AGR); 2) PN to afforestation with pines (MP); 3) Strips thinned

Recibido: 16 marzo 2018. Aceptado: 16 abril 2018. 
within MP: secondary grassland (PS). Soil samples were taken from each system, and both chemical and biochemical properties were analyzed. All the systems recorded a reduction of the $\mathrm{pH}$ and an increase in the $\mathrm{H}^{+}$saturation of the soil. An increase in the total organic carbon (COT) was observed in MP and PS. The anaerobic N mineralization potential (Nan) of these sites was much lower than that of PN, probably due to differences in the quality of their organic matter. PS generated a decrease in soil acidity, and an increase in Nan and P extractable. Acidification was lower in AGR, but it recorded the highest reduction in COT, Nan and $\mathrm{P}$ extractable. The changes observed indicate that the original characteristics of the soil tend to recover quickly in places where introduced vegetation was replaced..

Key words: Acidity, soil carbon, silvopastoral systems.

\section{INTRODUCCIÓN}

El sudeste de la provincia de Buenos Aires (SEB), es una región argentina de gran importancia productiva. El clima de la zona es templado húmedo sin estación seca $\left(\mathrm{C}_{\mathrm{fb}}\right.$, según Köppen). Las temperaturas máximas, mínimas $\mathrm{y}$ medias diarias presentan valores promedio anuales de $19,7,14,1$ y $8,4^{\circ} \mathrm{C}$, respectivamente. La precipitación media acumulada anual para el período 1971-2015, fue de $924 \mathrm{~mm}$. Los suelos de la zona se desarrollaron a partir de depósitos eólicos del período cuaternario esencialmente limosos (loess), y evolucionaron bajo vegetación de pradera. El resultado de la combinación de material madre, clima y vegetación produjo suelos Argiudoles que presentan en su condición original alta fertilidad y productividad, alto contenido de materia orgánica $(\mathrm{MO})$ y textura generalmente franca en superficie (Durán et al., 2011).

En las últimas tres décadas en el SEB, como en el resto de Argentina, se produjo un notable proceso de agriculturización, el cual incluyó la incorporación de nuevas áreas a la agricultura, la eliminación de las pasturas en las rotaciones y la simplificación de los esquemas de secuencias de cultivos (SAGPyA, 2006). Estos procesos dieron lugar al uso más intensivo de los suelos, a un exceso de laboreo (mayor cantidad de labores y más agresivas) e inoportuno (Darwich, 1991). También se produjo reemplazo de vegetación nativa de pastizales naturales por agricultura, mientras que en los piedemontes serranos, algunos pastizales fueron reemplazados por forestaciones, debido a las restricciones de estos sitios para la agricultura (Durán et al., 2011). Otro cambio implementado en algunos sitios, fue la reconversión de forestaciones a sistemas silvopastoriles (SSP), mediante el raleo de franjas de árboles en las mismas. Estos cambios en la vegetación, asociados a cambios en el manejo del suelo, tienen un efecto directo en las propiedades químicas y bioquímicas de los mismos (Durán et al., 2011).
Una de las propiedades más afectada por los cambios en el uso de la tierra, es el carbono orgánico total (COT). En el SEB, la conversión de vegetación nativa a agricultura provoca reducciones en el contenido de COT de los suelos (Sainz Rozas et al., 2011; Rodríguez et al., 2015). Por otra parte, en el caso de la forestación de pastizales, se han encontrado tanto reducciones (Berthrong et al., 2009), como aumentos o ausencia de efectos sobre el COT (Fialho y Zinn, 2014).

La disminución del contenido de COT tiene como consecuencia una reducción en la capacidad de abastecimiento de nutrientes, como el nitrógeno $(\mathrm{N})$ y el fósforo $(\mathrm{P})$. El uso agrícola de los suelos provoca disminuciones en el potencial de mineralización de $\mathrm{N}$ en la capa arable, independientemente del sistema de labranza (Reussi Calvo et al., 2013). El mayor uso agrícola de los suelos, la baja reposición de $\mathrm{P}$, y el aumento en la frecuencia del cultivo de soja en la rotación provocaron una disminución en la concentración de P disponible en los suelos de la región (Sainz Rozas et al., 2012). En cuanto a la presencia de árboles se han reportado tanto aumentos (Alonso, 2011) como reducciones en la disponibilidad de N y P (Mead, 2009).

A su vez, las variaciones en el uso de la tierra generan cambios en el $\mathrm{pH}$ de los suelos, afectando tanto el proceso de mineralización como la disponibilidad de nutrientes (Castro Diez et al., 2012). El uso agrícola continuo del suelo genera disminución del $\mathrm{pH}$, debido a factores como el desbalance entre la extracción y la reposición de nutrientes básicos (Ca, K, Mg) (Vázquez, 2005), el uso de siembra directa y de fertilizantes de reacción ácida (Divito et al., 2011, Wyngaardet al., 2012). Las forestaciones también pueden ocasionar acidez en los suelos debido a la alta extracción de cationes (Grubay Mulder, 2015), estando condicionado el proceso por la especie arbórea en cuestión (Castro Diez et al., 2012).

Para poder realizar un correcto uso de los recursos, resulta clave la comprensión de los procesos que ocurren en el suelo luego de 
un cambio en la vegetación. Son reconocidos los efectos del reemplazo de pastizales por agricultura, sin embargo, los efectos del reemplazo de pastizales por forestaciones, así como de la apertura del dosel arbóreo para reconversión a SSP han sido menos evaluados, particularmente en zonas templadas.

El objetivo de este trabajo fue estudiar los cambios en algunas propiedades del suelo generados por el cambio en el uso de la tierra desde pastizal natural a agricultura y forestación con pinos, y franjas raleadas en la forestación para implementar sistemas silvopastoriles.

\section{MATERIALES Y MÉTODOS}

Se seleccionaron cuatro sistemas de manejo establecidos sobre suelos con características físico-químicas similares (Tabla 1), ubicados en un establecimiento agropecuario en Tandil,

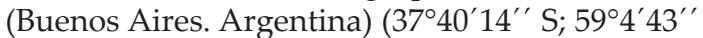
$W)$, con el objetivo de realizar un muestreo que permitiera estudiar el efecto de cambio en el uso de la tierra sobre variables edáficas.

El primer sistema seleccionado fue una forestación de pinos (Pinus radiata D. Don) de 22 años de antigüedad que se encuentra sobre un mosaico de suelos Argiudol Típico y Argiudol Vértico (Soil Survey Staff, 2014), pertenecientes a las series Tandil y Tres Esquinas, respectivamente (INTA, 2014). En los años 2011 y 2012 (tres y dos años antes del presente estudio), con el objetivo de realizar manejo silvopastoril, en esta forestación se eliminaron franjas de árboles de $10 \mathrm{~m}$ de ancho, en las que se permitió el crecimiento de especies nativas o naturalizadas (PS: pastizal secundario), dejando franjas de árboles separadas entre sí por 20 m (MP: monte de pinos). A fin de comparar el efecto de los manejos forestales con los manejos agrícolas habituales en la zona y con la situación prístina, se seleccionó un área adyacente bajo manejo agrícola con siembra directa (SD) por más de 20 años (AGR: agricultura). El suelo predominante en un $70 \%$ del área seleccionada es Argiudol Típico (Soil Survey Staff, 2014) serie Tandil (INTA, 2014), mientras que en el $30 \%$ restante se encuentra un suelo Argiudol Petrocálcico (Soil Survey Staff, 2014) de la serie Azul (INTA, 2014). El último sistema de manejo estudiado fue un área de pastizal natural (PN: pastizal natural) ubicado sobre un suelo similar, pero con algunas limitaciones por la presencia de roca aflorante. En este sitio alternan afloramientos rocosos, con suelo Hapludol Lítico (Soil Survey Staff, 2014), perteneciente a la serie Sierra de los Padres (INTA, 2014). Cada sistema de manejo estaba representado por un área de entre 8 y 12 ha.

Tabla 1. Descripción de los suelos en sitios bajo pastizal natural (PN), monte de pinos (MP), pastizal secundario (PS) y agricultura (AGR).

Table 1. Description of soils under natural grassland (PN), pine forest (MP), secondary grassland (PS) and agriculture (AGR).

\begin{tabular}{|c|c|c|c|c|}
\hline Sitio & PN & MP & PS & AGR \\
\hline Serie & $\begin{array}{l}\text { Sierra de } \\
\text { los Padres }\end{array}$ & $\begin{array}{l}\text { Tandil-Tres } \\
\text { esquinas }\end{array}$ & $\begin{array}{l}\text { Tandil-Tres } \\
\text { esquinas }\end{array}$ & Tandil-Azul \\
\hline Clasificación & $\begin{array}{l}\text { Hapludol } \\
\text { Lítico }\end{array}$ & $\begin{array}{l}\text { Argüidor } \\
\text { Típico- } \\
\text { Argiudol } \\
\text { Vértico }\end{array}$ & $\begin{array}{l}\text { Argiudol } \\
\text { Típico- } \\
\text { Argiudol } \\
\text { Vértico }\end{array}$ & $\begin{array}{c}\text { Argiudol } \\
\text { Típico- } \\
\text { Argiudolpetrocálcico }\end{array}$ \\
\hline $\begin{array}{l}\text { Años de agricultura } \\
\text { en SD }\end{array}$ & - & - & - & $>20$ \\
\hline Rotación & - & - & - & Girasol/Soja/Trigo-Soja \\
\hline C ( $\left(\mathrm{g} \mathrm{kg}^{-1}\right.$ suelo $)$ & 0,88 & $0,39-0,43^{*}$ & $0,39-0,43^{*}$ & $0,39-0,44^{*}$ \\
\hline $\mathrm{N}$ ( $\mathrm{g} \mathrm{kg}^{-1}$ suelo $)$ & 0,074 & $0,032-0,03^{*}$ & $0,032-0,03^{*}$ & $0,032-0,03^{*}$ \\
\hline$\%$ As & 32,8 & $29,4-30,7^{*}$ & $29,4-30,7^{*}$ & $29,4-30,3^{*}$ \\
\hline $\mathrm{pH}(1)$ & 6,1 & $6,9-6,7^{*}$ & $6,9-6,7^{*}$ & $6,9-6,3^{*}$ \\
\hline Profundidad del Hz A & 15 & $20-30^{*}$ & $20-30^{*}$ & $20-20^{*}$ \\
\hline
\end{tabular}

Nota: Los datos fueron extraídos de la carta de suelos del INTA (INTA, 2014). Los datos de C, N, \%As y pH (1), corresponden al horizonte A o Ap de cada suelo a la profundidad aquí analizada. ${ }^{*}$ Los valores en la celda corresponden a la primera y segunda serie de suelo mencionada, respectivamente.

Note: Data were obtained from INTA's soil chart (INTA, 2014). Data of C, N, \% As and pH (1) correspond to the horizon A or Ap of each soil at the soil depth analyzed. *Cell values correspond to the first and second soil series mentioned, respectively. 
En la Tabla 1 se presenta una descripción de los suelos de cada sitio. Es importante mencionar que los cuatro sitios analizados se encuentran distanciados entre sí no más de 300 metros, por lo que están sometidos a las mismas condiciones climáticas. La heterogeneidad del relieve en la zona fundamenta que en distancias tan cortas se presenten varias series de suelo, aunque todas provienen del mismo material parental (Loess). Se destaca que los mismos presentan valores similares de arcilla, entre 30 y $38 \%$ y de COT, entre 39 y $43 \mathrm{~g} \mathrm{~kg}^{-1}$ suelo en los primeros $20 \mathrm{~cm}$ del perfil (profundidad que se analizó en este estudio), a excepción de PN, cuyo COT es aproximadamente el doble que el del resto de los sitios. La gran similitud entre las características de suelo del estrato del perfil analizado entre los cuatro sitios, hace posible su comparación.

\section{Muestreo, acondicionamiento y análisis de suelo}

Se realizaron muestreos de suelo en las cuatro situaciones seleccionadas (MP, PS, PN y AGR), a dos profundidades: 0-5 y 5-20 $\mathrm{cm}$. Se tomaron aleatoriamente tres muestras compuestas de al menos 20 sub-muestras en cada situación, siguiendo transectas con muestreos cada 50 pasos de manera de lograr representatividad en cada sitio. Previo a la toma de cada muestra se eliminaron los residuos vegetales presentes en superficie, de modo que todos los datos aquí presentados corresponden a horizontes minerales. Todas las muestras fueron secadas en estufa a $30^{\circ} \mathrm{C}$, molidas y tamizadas con tamiz de $2 \mathrm{~mm}$ o $0,5 \mathrm{~mm}$, según el análisis a realizar. En las muestras de cada sistema de manejo se realizaron las siguientes determinaciones: acidez activa (1:2,5 en agua, Dewis y Freitas, 1970), acidez intercambiable (Thomas, 1982), contenido de carbono orgánico total (COT) mediante combustión húmeda con mantenimiento de la temperatura de reacción $\left(120^{\circ} \mathrm{C}\right.$, durante $\left.90 \mathrm{~min}\right)$ (Schlichting et al., 1995), P extractable Bray (Bray y Kurtz, 1945), N potencialmente mineralizable por incubación anaeróbica (Nan) (Waring y Bremner, 1964), capacidad de intercambio catiónico (CIC) (Summer y Miller, 1966), y conductividad eléctrica (Rhoades, 1996).

\section{Análisis estadístico}

Los datos fueron analizados mediante el software estadístico R (R Core Team, 2010). Se realizaron análisis de la varianza (ANOVA), para todas las variables estudiadas, separadamente para cada profundidad. Cuando se hallaron diferencias entre sitios, las comparaciones entre medias se realizaron mediante el test de mínima diferencia significativa de Fischer (LSD), a un nivel de significancia del 5\%.

\section{RESULTADOS Y DISCUSIÓN}

\section{Acidez del suelo}

La acidez activa del suelo $(\mathrm{pH})$ presentó diferencias significativas en respuesta al sistema de manejo, la profundidad de muestreo y la interacción entre ellos (Fig. 1A) ( $p<0,05)$. En todos los sistemas de manejo estudiados se redujo el $\mathrm{pH}$ en relación a la situación original (PN). De igual forma, el análisis de la acidez intercambiable del suelo mostró diferencias significativas entre sitios, profundidades e interacción entre ellos $(\alpha<0,05)$. En concordancia con las variaciones en el $\mathrm{pH}$, se encontró que MP, PS y en menor medida AGR, produjeron un aumento de la acidez intercambiable del suelo con respecto a PN (Fig. 1.B). Por otro lado, no se verificó presencia de $\mathrm{Al}$ intercambiable en ninguno de los sitios, por lo que se puede afirmar que las variaciones encontradas son debidas a reemplazo de bases intercambiables $\left(\mathrm{Ca}^{+2} \mathrm{y} \mathrm{Mg}^{+2}\right)$ por protones $\left(\mathrm{H}^{+}\right)$.

La mayor acidificación se observó en MP a ambas profundidades. Luego de 22 años desde el reemplazo del pastizal por la forestación, el suelo redujo su $\mathrm{pH} 0,64$ unidades y aumentó su saturación con $\mathrm{H}^{+} 246 \%$ (promedio de ambas profundidades). Este comportamiento se puede atribuir a la combinación de varios procesos que interactúan en el ecosistema forestal. Entre estos procesos se encuentran variaciones en el $\mathrm{pH}$ del agua de lluvia al atravesar la copa de los árboles, variaciones microclimáticas, producción de ácido carbónico, cantidad y composición del mantillo incorporado al suelo y balance de cargas producidas por la absorción de nutrientes (Amiotti et al., 2011). Además, la composición química del mantillo incorporado al suelo tiene bajo contenido de $\mathrm{Ca}$ y presencia de compuestos orgánicos complejos como lignina lo cual inhibe la actividad de la fauna, favoreciendo la flora fungosa. Este cambio de organismos descomponedores tiene consecuencias en los productos que se forman durante la descomposición, siendo el efecto final, una disminución marcada del $\mathrm{pH}$ (FalkengrenGrerup et al., 2006, Castro Diez et al., 2012). Se ha reportado que acompañando a los efectos sobre el $\mathrm{pH}$, las forestaciones redujeron la saturación del complejo de intercambio con bases a tres cuartos del valor original (del $59 \%$ al $45 \%$ ) a partir de disminuciones en la fracción intercambiable de $\mathrm{Mg}^{++}, \mathrm{K}^{+}, \mathrm{y} \mathrm{Ca}^{++}$(Jobaggy et al., 2006; Gruba y Mulder, 2015). Estos sitios serían posteriormente reemplazados por $\mathrm{H}^{+}$intercambiable, explicando el notorio aumento de dicho valor.

Cuando MP se reconvirtió a PS, la eliminación de la influencia directa de los residuos de pinos originó cambios en el pH y en la saturación con $\mathrm{H}^{+}$. Luego de tres años desde la reconversión del 

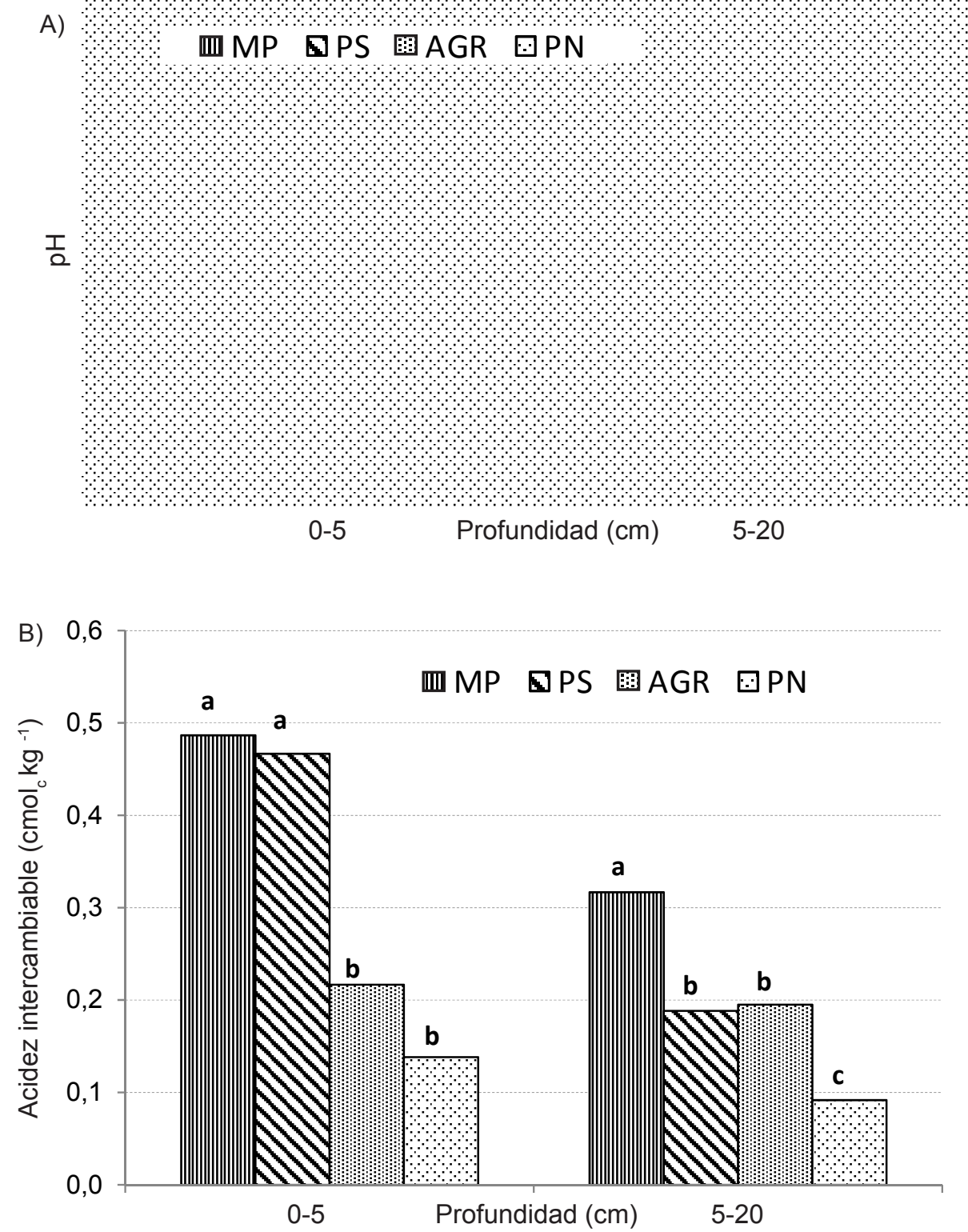

Fig. 1. Acidez activa (A) e intercambiable (B) del suelo en diferentes situaciones de manejo: (MP: monte de pinos, PS: pastizal secundario luego del desmonte, AGR: agricultura bajo rotaciones y siembra directa y PN: pastizal natural) a dos profundidades de suelo $(0-5 \mathrm{~cm}$ y $5-20 \mathrm{~cm})$. Letras minúsculas diferentes indican diferencias significativas (LSD, $\alpha=0,05$ ).

Fig. 1. Active (A) and exchangeable (B) soil acidity (B) under different soil management systems (MP: pine forest; PS: secondary grassland after clearing; AGR: agriculture under rotations and no tillage; PN: natural grassland) at two different soil depths $(0-5 \mathrm{~cm}$ and $5-20 \mathrm{~cm})$. Different lowercase letters indicate significant differences (LSD, $\alpha=0.05$ ).

sistema, PS presentó un aumento significativo del $\mathrm{pH}$ de 0,21 y 0,2 unidades de $\mathrm{pH}$ a 0-5 y $5-20 \mathrm{~cm}$, respectivamente (Fig. 1. A). La saturación con $\mathrm{H}^{+}$ de PS, en los primeros $5 \mathrm{~cm}$ del suelo no difirió de MP, mientras que en $5-20 \mathrm{~cm}$ de profundidad se diferenció de aquél, reduciendo su valor casi a la mitad (Fig. 1. B) $(\alpha<0,05)$. No se esperaba encontrar este resultado, ya que se pensaba que el mayor cambio ocurriría en los primeros $\mathrm{cm}$ del suelo. No obstante, al momento del muestreo, las franjas raleadas aún presentaban una importante cantidad de mantillo de pinos en estado parcial 
de descomposición, y además seguían recibiendo aporte de hojas desde las franjas de árboles contiguas. En este estrato del perfil, entonces sería más lento el proceso de recuperación de la saturación con bases, por lo cual en el período analizado, el cambio se observa sólo en el $\mathrm{pH}$. Se hipotetiza que las variaciones en acidez y $\mathrm{pH}$ en estratos más profundos del perfil son debidas en mayor medida, a la gran extracción de bases que los árboles realizan (Jobaggyet al., 2006), y no tanto a la influencia directa del residuo y los productos de su descomposición, como puede ocurrir en superficie (Falkengren-Grerup et al., 2006, Castro Diez et al., 2012). Así, tanto el $\mathrm{pH}$ como la saturación con $\mathrm{H}^{+}$se recuperarían más rápidamente en el estrato $5-20 \mathrm{~cm}$, ya que la eliminación de los árboles genera un efecto instantáneo de reducción en la extracción de cationes, recuperándose el suelo con más facilidad.

La acidificación ocasionada por el manejo agrícola fue menor que la observada en MP y PS. El pH de suelo se redujo 0,31 unidades en relación al PN, en coincidencia con la tendencia general encontrada para la Región Pampeana de 0,29 unidades de $\mathrm{pH}$ (Sainz Rozas et al., 2011). Se encontró también, un aumento significativo en la saturación con $\mathrm{H}^{+}$de $56 \%$ y $112 \%$ en $0-5$ y $5-20$ $\mathrm{cm}$, respectivamente. El proceso de acidificación se asocia a la intensificación en el uso de los suelos ocurrida durante las últimas décadas, con un aumento en la utilización de fertilizantes nitrogenados de reacción ácida, implementación de labranzas reducidas o siembra directa (Wyngaard et al., 2012) y falta de reposición de nutrientes, principalmente Ca y Mg (Vázquez, 2005). Bajo uso agrícola se verificó que el proceso de acidificación fue más intenso en los estratos superficiales (0,12 unidades) (Fig. 1. A y 1. B), lo cual puede deberse a que es en superficie donde se aplican los fertilizantes (Divito et al., 2011).

Los suelos estudiados se caracterizan por poseer arcillas de tipo 2:1, fundamentalmente illita y montmorillonita, y alto contenido de materia orgánica, lo que les confiere un alto poder amortiguador (Vázquez, 2005). Bajo estas condiciones, las variaciones de $\mathrm{pH}$ y particularmente de $\mathrm{H}^{+}$halladas cobran mayor relevancia. A pesar que las variaciones del $\mathrm{pH}$ no son grandes, el aumento en la saturación con $\mathrm{H}^{+}$implica disminución de la reserva de los nutrientes básicos, así como posibilidad de desbalances entre los mismos, lo cual es también perjudicial para el crecimiento de las plantas (Vázquez, 2005).

\section{Conductividad eléctrica}

En los primeros $5 \mathrm{~cm}$ del perfil, todos los sistemas de manejo produjeron un aumento en la conductividad eléctrica (CE) con respecto a PN. En los sitios MP y PS, dicho aumento ocurrió también en profundidad, mientras que en AGR, este efecto se verificó sólo en superficie (Fig. 2).

Jackson et al. (2005) estudiaron transectas de suelo bajo forestaciones, praderas y/o manejo agrícola, y encontraron que en la mayoría de los casos la CE era superior en suelos de montes con respecto a los otros sistemas de manejo. Los autores reportaron relaciones $\mathrm{CE}_{\text {forestación }}: \mathrm{CE}_{\text {pradera }}$ entre 0,7 y 29,8, independientes de la especie arbórea en cuestión, y en mayor medida en suelos de textura franca similares a los de este estudio. Además observaron que la implantación de forestaciones sobre praderas, produjo un aumento significativo de la concentración de sodio en solución e intercambiable, y disminución de la saturación con bases, lo que sugiere salinización y/o sodificación (Jobbagyy Jackson, 2004). Los autores atribuyeron este patrón a un efecto combinado de aumento de la evapotranspiración bajo influencia forestal y el consecuente ascenso de sodio presente en la napa. En el caso del suelo en estudio, la relación $\mathrm{CE}_{\text {forestación }}: \mathrm{CE}_{\text {pradera }}$ fue 1,89 (promedio de ambas profundidades). A pesar de que este comportamiento está en línea con lo reportado por los mencionados autores, ninguno de los sitios estudiados presentó valores de CE mayores al valor establecido por USDA de $4 \mathrm{dS} \mathrm{m} \mathrm{m}^{-1}$, como límite para considerar un suelo salino. A diferencia de los suelos del mencionado estudio, en los sitios donde se desarrolló este trabajo, la concentración de $\mathrm{Na}^{+}$en el agua subterránea no es alto y la napa se encuentra a gran profundidad, por lo cual el efecto sería debido al aumento de la evapotranspiración. La media de evapotranspiración de referencia (ET0) para el período fue de $960 \mathrm{~mm}$ y seguramente fue mayor en el área forestada.

A diferencia de lo que ocurre con el $\mathrm{pH}$, en la Fig. 2 puede verse que la CE del pastizal secundario no se diferenció de la de MP que le dio origen $(p>0,05)$, sugiriendo que los procesos que llevan a un aumento de la CE se revierten con mayor lentitud que los que modifican el $\mathrm{pH}$, o que las franjas de árboles sin eliminar tienen un efecto sobre el suelo de los espacios abiertos entre ellas. Se encontró además que PS presenta una tendencia inversa a lo esperado, ya que su CE fue significativamente mayor a la profundidad de $5-20 \mathrm{~cm}$ que a $0-5 \mathrm{~cm}$. No se han encontrado publicaciones con resultados similares, por lo cual se necesitaría realizar un seguimiento en el tiempo de esta variable.

El sitio AGR presentó valores de CE en 


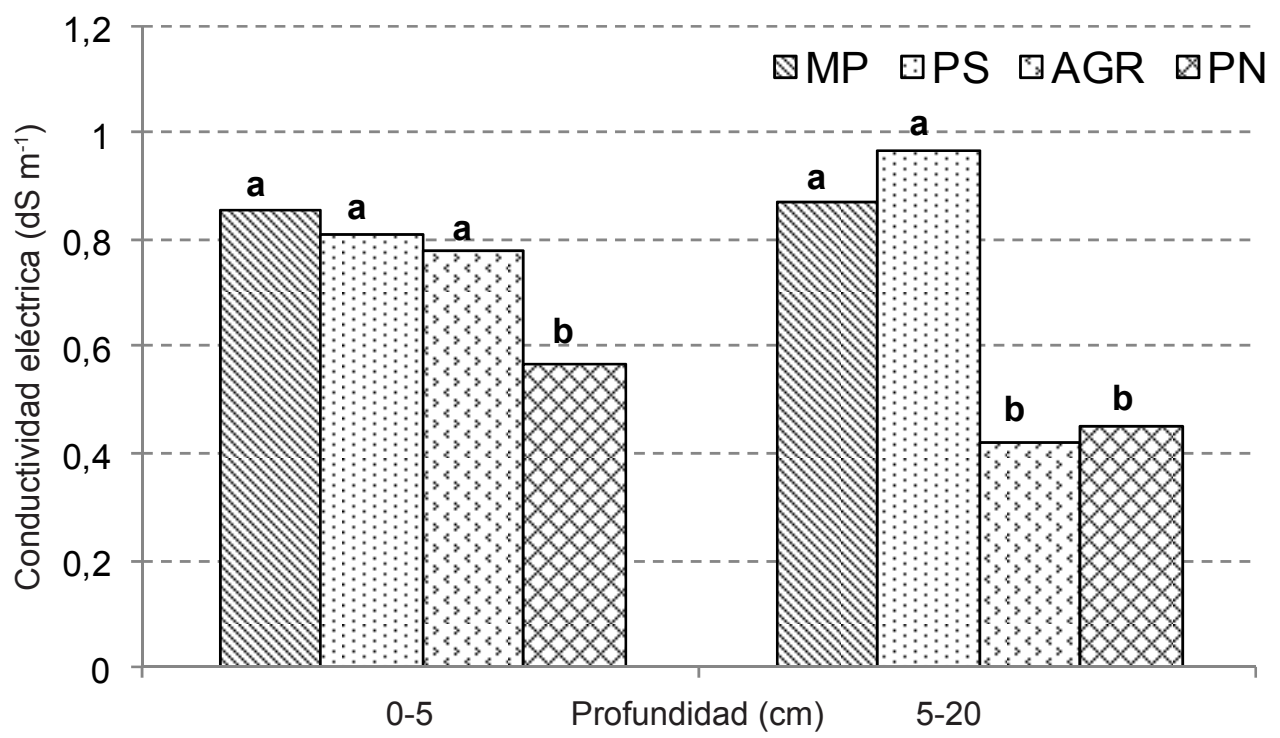

Fig. 2. Conductividad eléctrica en diferentes situaciones de manejo (MP: monte de pinos, PS: pastizal secundario luego del desmonte, AGR: agricultura bajo rotaciones y siembra directa y PN: pastizal natural) a dos profundidades de suelo $(0-5 \mathrm{~cm}$ y 5-20 cm). Letras minúsculas diferentes indican diferencias significativas (LSD, $\alpha=0,05$ ).

Fig. 2. Soil electrical conductivity under different soil management systems (MP: pine forest; PS: secondary grassland after clearing; AGR: agriculture under rotations and no tillage; and PN: natural grassland) at two different soil depths $(0-5 \mathrm{~cm}$ and 5-20 cm). Different lowercase letters indicate significant differences (LSD, $\alpha=0.05$ ).

superficie similares a MP y PS, pero este comportamiento no se mantuvo en profundidad (Fig. 2). En cambio en $5-20 \mathrm{~cm}$, no presentó diferencias con la situación sin disturbar (PN). Esto sugiere que los procesos que causan el aumento de la CE en el manejo agrícola ocurren solo en los primeros $\mathrm{cm}$ del suelo, debido por ejemplo, a la aplicación de fertilizantes en superficie. Sin embargo, en ninguno de los sitios estudiados se superó el umbral de salinidad de $4 \mathrm{dS} \mathrm{m}^{-1}$ (USDA, 1996). Es decir, que hasta el momento del muestreo, los cambios en contenido salino no afectarían el normal desarrollo de los cultivos utilizados en la zona.

\section{Carbono orgánico total}

ElCOT de los sitios analizados presentó valores entre 0,83 y $0,25 \mathrm{~g} \mathrm{~kg}^{-1}$ de suelo, y fue afectado significativamente por el sitio, la profundidad de muestreo y la interacción entre dichas variables $(p<0,05)$. Tal como se esperaba, el COT de todos los sitios fue superior en los primeros $5 \mathrm{~cm}$ del suelo $\left(0,69 \mathrm{~g} \mathrm{~kg}^{-1}\right.$ suelo en promedio) con respecto al estrato 5-20 cm (0,42 $\mathrm{g} \mathrm{kg}^{-1}$ suelo en promedio). Independientemente de la profundidad, la mayor acumulación de carbono se observó en MP y la menor en AGR ( $p<0,05)$. En los primeros $5 \mathrm{~cm}$ de suelo el COT de PN fue $0,71 \mathrm{~g} \mathrm{~kg}^{-1}$ de suelo (Fig. 3), valor habitual en pastizales naturales de la zona (Rodríguez et al., 2015). Los sitios MP y PS presentaron los mayores valores $\left(0,82 \mathrm{~g} \mathrm{~kg}^{-1} \mathrm{de}\right.$ suelo, en promedio), superior a lo comunicado por Eclesia et al. (2012) en forestaciones de zonas templadas del continente americano. El COT fue 38\%, 32\%, $45 \%$ y 36\% menor en el estrato 5-20 cm con respecto al estrato $0-5 \mathrm{~cm}$, respectivamente para $\mathrm{PN}, \mathrm{MP}, \mathrm{PS}$ y AGR, sin diferencias significativas entre PN y PS en esta última profundidad $(\mathrm{p}<0,05)$.

El reemplazo de $\mathrm{PN}$ por MP produjo aumentos significativos en el contenido de COT (Fig. 3) de 0,123 y $0,131 \mathrm{~g} \mathrm{~kg}^{-1} \mathrm{de}$ suelo a las profundidades de 0-5 y 5-20 cm, respectivamente, superiores a los comunicados para suelos Argiudoles de la misma región geográfica cuando se remplazó vegetación nativa por forestaciones con acacias (Rodríguez et al., 2015). A pesar de que los pastizales naturales son la vegetación original de la zona estudiada, según nuestros resultados el sitio $\mathrm{PN}$ aún no ha alcanzado la saturación con C. En coincidencia con nuestros resultados, Eclesia et al. (2012) concluyeron que el balance de carbono del suelo en las transiciones de vegetación natural a forestaciones, depende de la precipitación media 


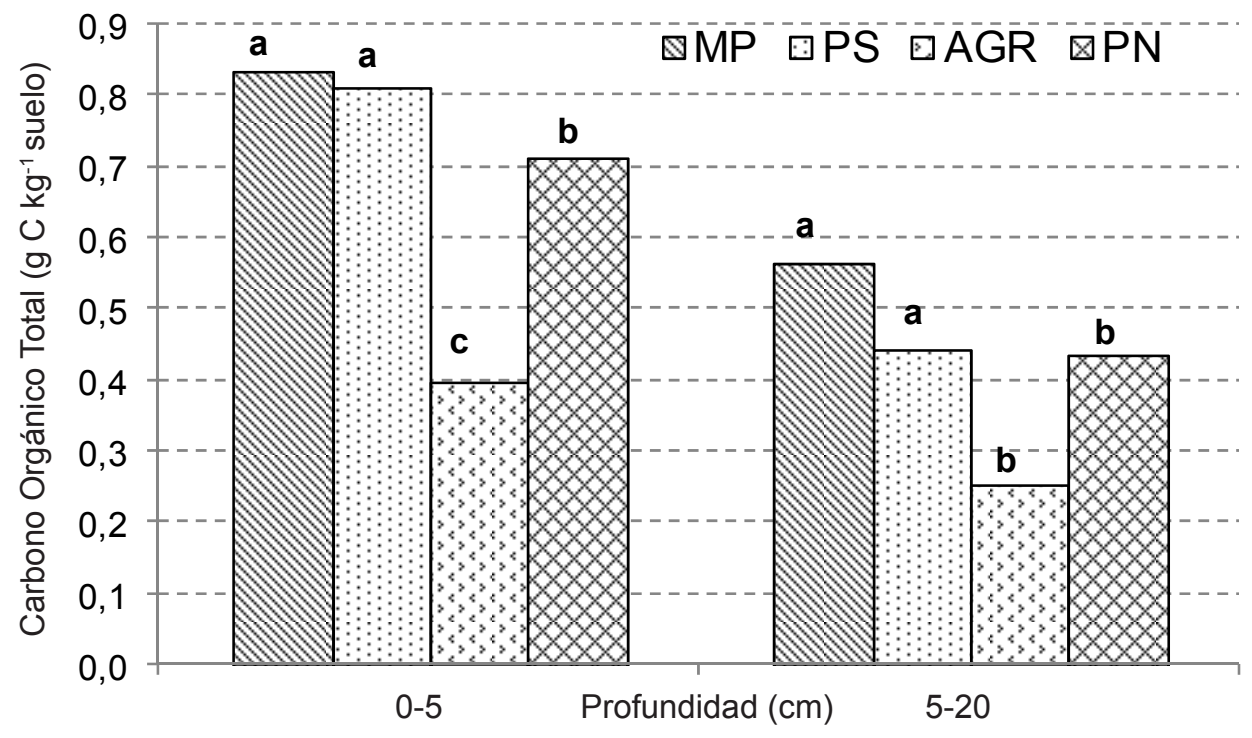

Fig. 3. Carbono orgánico total para diferentes situaciones de manejo (MP: monte de pinos, PS: pastizal secundario luego del desmonte, AGR: agricultura bajo rotaciones y siembra directa y PN: pastizal natural), a dos profundidades $(0-5 \mathrm{~cm}$ y $5-20 \mathrm{~cm})$. Letras minúsculas diferentes indicant diferencias significativas (LSD, $\alpha=0,05$ ).

Fig. 3. Soil organic carbon under different soil management systems (MP: pine forest; PS: secondary grassland after clearing; AGR: agriculture under rotations and no tillage; and PN: natural grassland) at two different soil depths $(0-5 \mathrm{~cm}$ and 5-20 cm). Different lowercase letters indicate significant differences (LSD, $\alpha=0.05$ ).

anual del sitio (PMA) y de la edad de la plantación. Así, los sitios con PMA menor a $1200 \mathrm{~mm}$ y más de 20 años desde la conversión, presentan ganancias netas de carbono o valores similares al de la vegetación original, lo cual coincide con las características de los sitios analizados en el presente estudio, ya que la precipitación media anual es de $950 \mathrm{~mm}$ (Estación Meteorológica de la EEA INTA Balcarce). Además, la relativamente baja tasa de descomposición de los residuos en las forestaciones contribuiría a incrementar el contenido de COT del suelo superficial, con respecto a los pastizales (Jobaggy y Jackson, 2000). El elevado contenido de $C$ en el suelo del ecosistema forestal $\left(0,686 \mathrm{~g} \mathrm{~kg}\right.$ de suelo ${ }^{-1}$, promedio de ambas profundidades), sería consecuencia de la gran cantidad de residuos aportados por los árboles, su alta relación $\mathrm{C} / \mathrm{N}$ $\mathrm{y}$ alto contenido de fracciones recalcitrantes que limitan su descomposición (FalkengrenGrerup et al., 2006, Castro Diez et al., 2012). Por otra parte, se considera que el carbono orgánico disuelto (COD) es una fuente importante de carbono en suelos forestales. Se ha reportado que entre 10 y $40 \mathrm{~g}_{\text {de }} \mathrm{COD} \mathrm{\textrm {m } ^ { 2 }}$ año ${ }^{-1}$ se traslocan desde los horizontes orgánicos a los horizontes minerales, mientras que los flujos se reducen a entre 1 y $10 \mathrm{~g}$ de COD m${ }^{2}$ año-1 en horizontes más profundos, sugiriendo una fuerte retención de COD en horizontes subsuperficiales (Michalzik et al., 2001). Al estimar la contribución potencial del $\mathrm{COD}$ al $\mathrm{C}$ total en el suelo mineral se encontró que el C del suelo proveniente del COD oscila entre 20 y $55 \mathrm{Mg} \mathrm{ha}^{-1}$ en el suelo mineral, lo que representa del 19\% al 50\% del COT (Kalbitz y Kaiser, 2008). Por lo expuesto, el COD podría ser una importante fuente de carbono que contribuiría a explicar los altos niveles de COT hallados en la forestación estudiada.La apertura del dosel arbóreo (PS) no generó pérdidas significativas de COT en los primeros $\mathrm{cm}$ del suelo en el período analizado (con respecto a MP). Sin embargo, en el estrato 5-20 cm, el COT de PS se redujo en 0,123 $\mathrm{g} \mathrm{kg}^{-1} \mathrm{de}$ suelo en relación a MP. Esto pone de manifiesto que la acumulación de $\mathrm{C}$ a dicha profundidad está directamente asociada a la presencia de los árboles.

El manejo agrícola (AGR) presentó menores valores de COT con respecto a los demás sistemas (Fig. 3). La reducción con respecto a PN fue de 0,31 y 0,18 $\mathrm{g} \mathrm{kg}^{-1}$ suelo para 0-5 y 5-20 cm, respectivamente, indicando que si bien actualmente el sistema es manejado bajo SD, los años previos de LC habrían producido una 
reducción de compuestos orgánicos debido a la oxidación generada por el laboreo (Sainz Rozas et al., 2011). En la Región Pampeana, los aportes de residuos de los cultivos anuales representan entre 30 y $70 \%$ de los atribuidos a los pastizales naturales, explicando parte de la disminución de los niveles de COT de los suelos cultivados en comparación con los no cultivados (Alvarez y Steinbach, 2012).La reducción de materia orgánica de los suelos del SEB, ya fue definido reportada por Sainz Rozas et al. (2011) fue de $36,6 \%$ en los primeros $20 \mathrm{~cm}$ del suelo con respecto a su situación prístina, lo cual es algo menor que los valores encontrados en este estudio (43,8\%, promedio ponderado de $0-20 \mathrm{~cm}$ ).

Acompañando los cambios en el contenido de COT, los cambios en el uso de la tierra estudiados produjeron variaciones significativas en la CIC del suelo. Esta variable presentó diferencias significativas entre sistemas de manejo y entre profundidades $(p<0,05)$, pero no del efecto combinado de estas variables (datos no mostrados). Se encontró que en los manejos donde crecieron pinos (MP y PS) la CIC fue mayor en relación al PN, mientras que bajo manejo agrícola (AGR) fue menor $(18,87 ; 21,69$; 16,95 y $14,63 \mathrm{cmol}_{\mathrm{c}} \mathrm{kg}^{-1}$ respectivamente). Por otra parte, la CIC en los primeros $5 \mathrm{~cm}$ de suelo $(18,5$ $\left.\mathrm{cmol}_{\mathrm{c}} \mathrm{kg}^{-1}\right)$ fue significativamente mayor que la de $5-20 \mathrm{~cm}\left(17,6 \mathrm{cmol}_{\mathrm{c}} \mathrm{kg}^{-1}\right)$.

La CIC es una propiedad química del suelo que depende de la cantidad y tipo de coloides presentes, tanto inorgánicos como orgánicos (MO). Los coloides orgánicos pueden tener una CIC de hasta $300 \mathrm{cmol} \mathrm{kg}^{-1}$ de suelo (Leinweberet al., 1993) y su contenido en el suelo es altamente dependiente del manejo. Sabiendo entonces que los cuatro manejos estudiados se ubicaron sobre suelos con un contenido de arcilla similar, se puede inferir que MP y PS tienen los mayores valores de CIC como consecuencia de su mayor contenido de COT, coincidente con lo reportado por Gruba y Mulder (2015). Pocos son los estudios en este tipo de ecosistemas en el SEB; Zalba y Peinemann (1987) compararon valores de CIC de suelos bajo varias especies arbóreas, entre ellas una plantación de Pinus radiata de 30 años y el suelo adyacente bajo vegetación natural herbácea, en zonas serranas de Ventania (sistema serrano contiguo a aquel en que se encuentran los sitios aquí estudiados). Estos autores encontraron valores significativamente mayores bajo el pinar que en el pastizal adyacente coincidiendo con lo encontrado en este trabajo.

\section{Nitrógeno pontencialmente mineralizable}

El análisis del Nan presentó diferencias significativas entre sitios, profundidades y la interacción entre dichas fuentes de variación ( $p$ $<0,05$ ). Esta variable presentó un amplio rango de valores entre 67,6 y $249,5 \mathrm{mg} \mathrm{N} \mathrm{kg}^{-1}$ de suelo (Fig. 4)

La condición de suelo inalterada (PN) presentó el mayor contenido de Nan con 249,5 mg N kg-1 suelo, para los primeros $5 \mathrm{~cm}$ (Fig. 4) similar a lo reportado para pastizales naturales de la Región Pampeana de 235,1 mg kg-1 (Rodríguez et al., 2015). Se observa que todos los manejos que modificaron la situación original condujeron a una reducción de la oferta de $\mathrm{N}$ mineralizable, principalmente en los primeros $5 \mathrm{~cm}$ de suelo, coincidiendo con otros autores (Fabrizzi et al., 2003; Videla et al, 2005; Rodríguez et al., 2015). A pesar de que MP y PS presentaron los valores más altos de COT, esto no se reflejó en el Nan; lo que puede deberse a una mayor relación $\mathrm{C} / \mathrm{N}$ de su materia orgánica (Dick et al., 2011) y a un mayor contenido de fracciones recalcitrantes que limitan la mineralización (Wiesmeier et al., 2009), ya que la capacidad de mineralización de $\mathrm{N}$ se relaciona fundamentalmente con el contenido de las fracciones lábiles (Reussi Calvo et al., 2013). En tal sentido, Di Gerónimo et al. (2018), trabajando en los mismos sitios aquí estudiados, encontraron que el mayor COT en MP y PS se explica por un mayor contenido de carbono en la fracción < $53 \mu \mathrm{m}$. En contraposición, PN contiene mayor proporción de $\mathrm{C}$ y $\mathrm{N}$ en la fracción $>53 \mu \mathrm{m}$. Esta última fracción, denominada materia orgánica particulada (MOP) representa una fuente de $\mathrm{C}$ y $\mathrm{N}$ de mayor labilidad en relación a aquella $<53$ $\mu \mathrm{m}$, explicando que en este caso, mayor COT no implica mayor potencial de mineralización de $\mathrm{N}$.

El manejo agrícola (AGR) presentó el valor más bajo de Nan, en las dos profundidades analizadas $(\mathrm{p}<0,05)$, indicando una mayor degradación del suelo en este sistema. Las disminución hallada con respecto a PN fue de 50,7\% (promedio de ambas profundidades) coincidente con lo reportado por Reussi Calvo et al. (2014), para suelos de la provincia de Buenos Aires. Es sabido que el uso agrícola de los suelos provoca reducción del contenido de materia orgánica, principalmente de fracciones lábiles. En este sitio se registró una disminución del COT (ya discutido) y del C y N en la MOP (Di Gerónimo et al., 2017), con respecto a PN, lo cual explica el menor Nan.

\section{Fósforo extractable (P-Bray)}

Se encontró efecto significativo del sistema de manejo, la profundidad y de la interacción entre dichos factores $(p<0,05)$ sobre la cantidad de P extractable. El manejo agrícola incrementó significativamente el valor de P-Bray respecto al resto de los tratamientos $\left(38,8 \mathrm{mg} \mathrm{kg}^{-1}\right.$ más en promedio) (Fig. 5). Esta gran diferencia se 


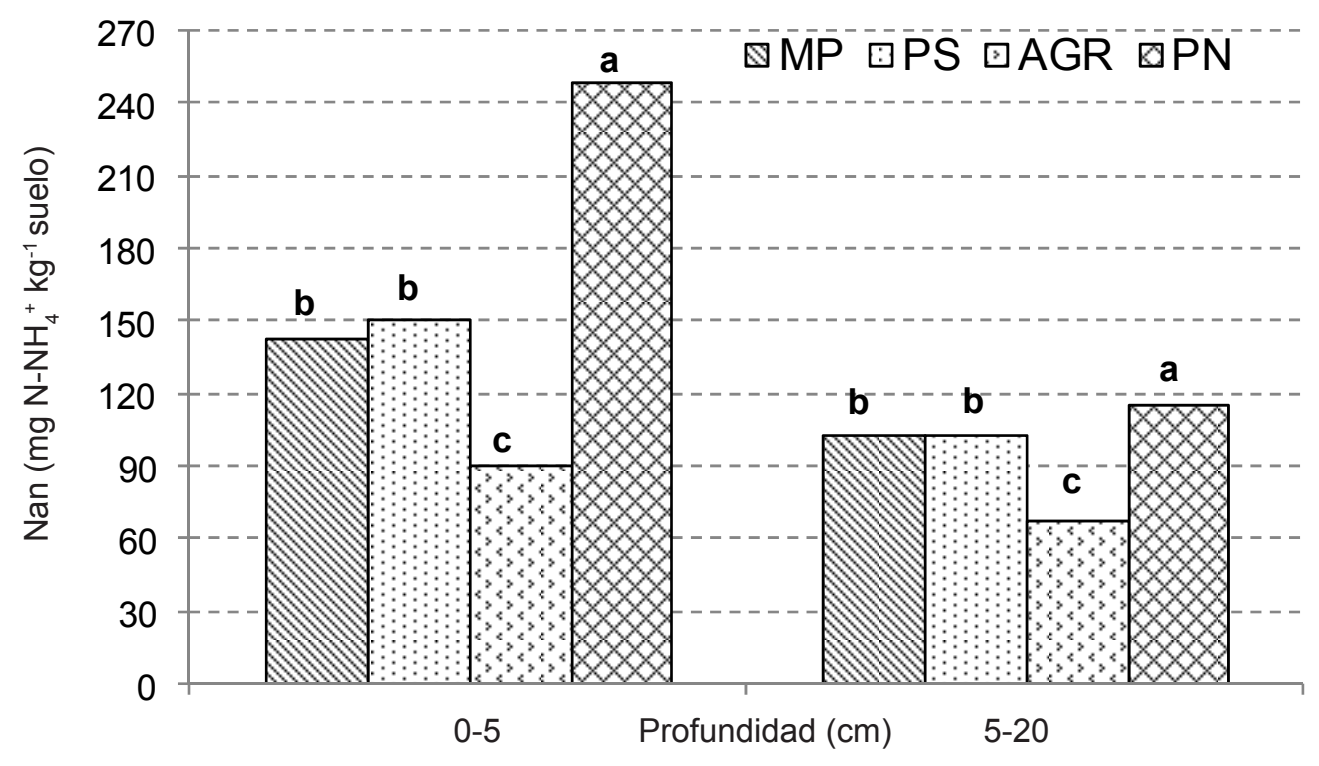

Fig. 4. Nitrógeno pontencialmente mineralizable estimado por incubación anaeróbica corta (Nan) para diferentes situaciones de manejo (MP: monte de pinos, PS: pastizal secundario luego del desmonte, AGR: agricultura bajo rotaciones y siembra directa y PN: pastizal natural) a dos profundidades de muestreo $(0-5 \mathrm{~cm}$ y $5-20 \mathrm{~cm}))$. Letras minúsculas diferentes indican diferencias significativas (LSD, $\alpha=0,05$ ).

Fig.4. Potentially mineralizable $\mathrm{N}$ estimated by short-term anaerobic incubation (Nan), under different soil management systems (MP: pine forest; PS: secondary grassland after clearing; AGR: agriculture under rotations and no tillage; and PN: natural grassland) at two different soil depths $(0-5 \mathrm{~cm}$ and 5-20 $\mathrm{cm})$. Different lowercase letters indicate significant differences (LSD, $\alpha=0.05$ ).

originaría en la continua fertilización fosfatada que ha recibido el sitio estudiado. Varias investigaciones han encontrado que las altas tasas de fertilización fosforada alteran el equilibrio natural del suelo aumentando significativamente la concentración de fosfato y el grado de saturación de P (Pose et al., 2012).

El reemplazo de PN por MP no produjo cambios significativos en el P-Bray en los primeros $5 \mathrm{~cm}$ del suelo, sin embargo, en el estrato $5-20 \mathrm{~cm}$ el valor de dicha variable fue superior en $6,06 \mathrm{mg}$ $\mathrm{kg}^{-1}$ suelo al de PN (Fig. 5). Se ha reportado que en suelos donde se ha introducido Pinus radiata como especie exótica, existe una disminución del $\mathrm{P}$ orgánico y del $\mathrm{P}$ total $(\mathrm{McD}$ cowell y Stewart, 2006) y aumentos de las fracciones fosfóricas inorgánicas, particularmente las disponibles medidas por el método de extracción de $\mathrm{P}$ utilizado en este trabajo (Mc Dowelly Stewart, 2006; Chen et al., 2008). Estudios previos en la zona también indican que existen importantes aportes de $\mathrm{P}$ provenientes de los horizontes orgánicos generados bajo P. radiata (Garay et al., 2012). Sin embargo, en los primeros $\mathrm{cm}$ del perfil, la disponibilidad del nutriente fue menor lo cual puede ser explicado por el efecto directo de la marcada acidez de este estrato sobre la disponibilidad de este nutriente (Frossard et al., 2000). En línea con esta teoría, el paso de MP a PS produjo un aumento de $9 \mathrm{mg} \mathrm{kg}^{-1}$ de suelo en los primeros $5 \mathrm{~cm}$, sin que se encontraran tales diferencias en el estrato $5-20 \mathrm{~cm}$, donde las variaciones en acidez fueron menores.

\section{CONCLUSIONES:}

La implantación de forestaciones con pino radiata en áreas serranas es una interesante herramienta de manejo que permite mantener o incluso mejorar los niveles de carbono del suelo. A pesar de la acidificación causada por los árboles, cuando se ralean franjas para uso silvopastoril el $\mathrm{pH}$ aumenta rápidamente generando mayor disponibilidad de nutrientes.

Los cambios observados son favorables para el desarrollo de sistemas silvopastoriles, los cuales permiten diversificar la producción, utilizando áreas del campo con restricciones para la agricultura. 


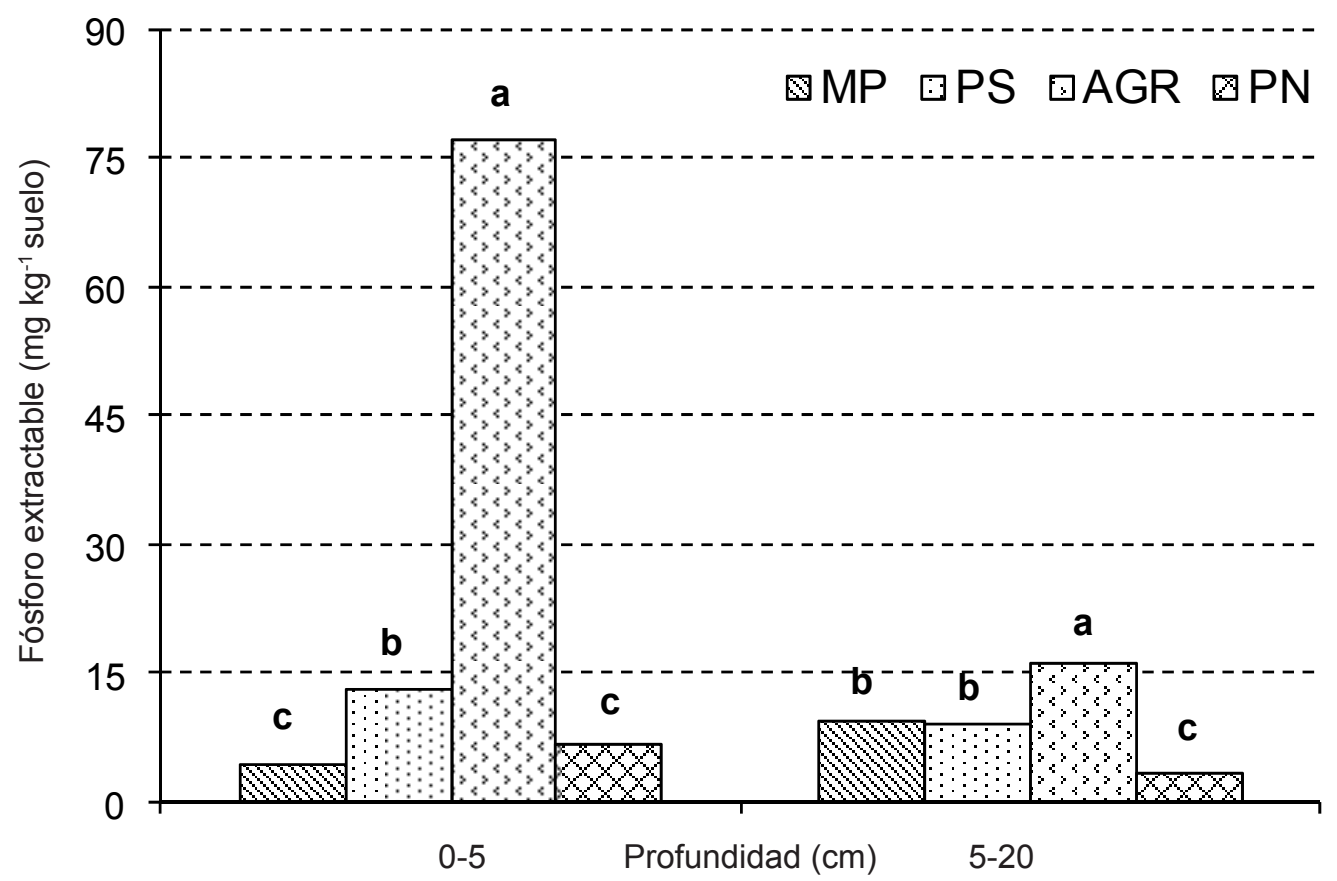

Fig. 5. Fósforo extractable para diferentes situaciones de manejo (MP: monte de pinos, PS: pastizal secundario luego del desmonte, AGR: agricultura bajo rotaciones y siembra directa y PN: pastizal natural) a dos profundidades de muestreo $(0-5 \mathrm{~cm}$ y $5-20 \mathrm{~cm})$. Letras minúsculas diferentes indican diferencias significativas (LSD, $\alpha=0,05$ ).

Fig. 5. Extractable phosphorus under different soil management systems (MP: pine forest; PS: secondary grassland after clearing; AGR: agriculture under rotations and no tillage; and PN: natural grassland) at two different soil depths $(0-5 \mathrm{~cm}$ and 5-20 cm). Different lowercase letters indicate significant differences (LSD, $\alpha=0.05$ ).

\section{LITERATURA CITADA}

Alonso, J. 2011. Los sistemas silvopastoriles y su contribución al medio ambiente. Revista Cubana de Ciencia Agrícola 45(2):107-115.

Alvarez, R., and H.S. Steinbach. 2012. Efecto del uso agrícola sobre el nivel de materia orgánica. p. 181-199. En R. Álvarez, G. Rubio, C.R. Álvarez, y R.S. Lavado (eds.). Fertilidad de suelos, caracterización y manejo en la Región Pampeana. Facultad de Agronomía, Universidad de Buenos Aires, Buenos Aires, Argentina.

Amiotti, N.M., P. Zalba, A. Ares, and J.M. Rossi. 2013. Coniferous afforestation increases soil carbon in maritime sand dunes.. Arch.Agron. Soil Sci. 59(2):289-304.

Berthrong, S.T., E.G. Jobbagy, and R.B. Jackson. 2011. A global meta-analysis of soil exchangeable cations, $\mathrm{pH}$, carbon, and nitrogen with afforestation. Ecol. Appl. 19(8):2228-2241.
Bray, R.H., and L.T. Kurtz. 1945. Determination of total, organic and available forms of phosphorus in soils. Soil Sci. 59(1):39-45.

Castro-Díez, P., N. Fierro-Brunnenmeister, N. González-Muñoz, and A. Gallardo. 2012. Effects of exotic and native tree leaf litter on soil properties of two contrasting sites in the Iberian Peninsula. Plant Soil 350(1,2):179-191

Chen, C., L. Condronand, and Z. Xu. 2008. Impacts of grassland afforestation with coniferous trees on soil phosphorus dynamics and associated microbial processes: a review. Forest Ecol. Manag. 255(3):396-409.

Chung H., J.H. Grove, and J. Six. 2008. Indications for soil carbon saturation in a temperate agroecosystem. Soil Sci. Soc. Am. J. 72(4):1132-1139.

Dewis, J., y F. Freitas. 1970. Métodos físicos y químicos de análisis de suelos y aguas. p. 3657. Boletín Sobre Suelos № 10. FAO, Roma, Italia. 
Di Gerónimo, P.F., C. Videla, and P. Laclau. 2018. Distribución de carbono y nitrógeno orgánico en fracciones granulométricas de suelos bajo pastizales, agricultura y forestaciones. Cienc. Suelo (Argentina) 36 (1): 11-22.

Dick, P.D., S.B. Leite, R.S. Diniz Dalmolin, H. Almeida, and H. Knicker. 2011. Pinus afforestation in South Brazilian highlands: soil chemical attributes and organic matter composition. Sci. Agr. 68(2):175-18.

Divito, G.A., H.R. Sainz Rozas, H.E. Echeverría, G.A. Studdert, and N. Wyngaard. 2011. Long term nitrogen fertilization: soil property changes in an Argentinean Pampas soil under no tillage. Soil Till. Res. 114(2):117-126.

Durán, A., H. Morrás, A. Studdert, and L. Xiaobing. 2011. Distribution, properties, land use and management of Mollisols in South America. Chin. Geogra. Sci. 21(5)511-530.

Eclesia R.P., E. Jobbagy, R.B. Jackson, F.B Biganzoli, and G. Piñeiro. 2012. Shifts in soil organic carbon for plantation and pasture establishment in native forests and grasslands of South America. Global Chan. Biol. 18(10):3237-3251

Fabrizzi, K.P., A. Morón, and F.O. García.2003. Soil carbon and nitrogen organic fractions in degraded vs. Non-degraded Mollisols in Argentina. Soil Sci. Soc. Am. J. 67(6):1831-1841.

Falkengren-Grerup, U., D.J. ten Brink, and J. Brunet. 2006. Land use effects on soil N, P, $\mathrm{C}$ and $\mathrm{pH}$ persist over $40-80$ years of forest growth on agricultural soils. Forest Ecol. Manag. 225:74-81.

Fialho, R.C., and Y.I. Zinn. 2014. Changes in soil organic carbon under eucalyptus plantations in Brazil: a comparative analysis. Land Degrad. Dev. 25(5):428-437.

Frossard, E., L. Condron, A. Oberson, S. Sinajand, and J. Fardeau. 2000. Processes governing phosphorus availability in temperate soils. J. Environ. Qual. 29(1):15-23.

Garay, M., N. Amiotti, and P. Zalba. 2012. Dinámica de nutrientes en rodales de Pinus radiata D. Don. En Actas $19^{\circ}$ Congreso Latinoamericano de la Ciencia del Suelo - $23^{\circ}$ Congreso Argentino de la Ciencia del Suelo. Abril 2012. [CD rom]. Asociación Argentina de la Ciencia del Suelo y Universidad Nacional de Mar del Plata. Mar del Plata, Buenos Aires, Argentina.

Graham, M.H., R.J. Haynes, and J.H. Meyer. 2002. Soil organic matter content and quality: effects of fertilizer applications, burning and trash retention on a long-term sugarcane experiment in South Africa. Soil Biol. Biochem. 34(1):93-102.
Gruba, P., and J. Mulder. 2015. Tree species affect cation exchange capacity (CEC) and cation binding properties of organic matter in acid forest soils. Sci. Total Environ. 511(1):655662.

Guo, L.B., and R.M. Gifford. 2002. Soil carbon stocks and land use change: a meta analysis. Glob. Change Biol. 8(4):345-360.

INTA. 2014. Cartas de suelos de la República Argentina -Provincia de Buenos Aires. Disponible en http://anterior.inta.gov.ar/ suelos/cartas/ (Consulta 05 febrero 2017).

Jackson, R.B., E.G. Jobbagy, R. Avissar, S. Baidya Roy, D.J. Barrett, C.W Cook, et al. 2005. Trading water for carbon with biological carbon sequestration. Science 310(5756):19441947.

Jobbagy, E.G., and R.B. Jackson. 2000. The vertical distribution of soil organic carbon and its relation to climate and vegetation. Ecol. Appl. 10(2):423-436.

Jobbagy, E.G., and R.B. Jackson. 2004. Groundwater use and salinization with grassland afforestation. Global Change Biol. 10(8):1299-1312.

Jobbagy, E.G., M. Vasallo, K.A. Farley, G. Piñeiro, M.F. Garbulsky, M.D. Nosetto, et al. 2006. Forestación en pastizales: Hacia una visión integral de sus oportunidades y costos ecológicos. Agrociencia 10(2):109-124.

Kalbitz, K., and K. Kaiser. 2008. Contribution of dissolved organic matter to carbon storage in forest mineral soils. J. Plant Nutr. Soil Sc. 171(1):52-60.

Lal, R. 2004. Soil carbon sequestration impacts on global climate change and food security Science 304(5677):1623-1627.

Leinweber, P., G. Reuter, and K. Brozio. 1993. Cation exchange capacity of organo-mineral particle size fractions in soils from long-term experiments. J. Soil Sci. 44:111-119.

McDowell, R., and I. Stewart. 2006. The phosphorus composition of contrasting soils in pastoral, native and forest management in Ontago, New Zealand: Sequential extraction and 31P NMR. Geoderma 130(1):176-189.

Mead, D. 2009. Biophysical interactions in silvopastoral systems: a New Zealand perspective. p. 3-9. En Actas $1^{0}$ Congreso Nacional de Sistemas Silvopastoriles. Mayo 2009. Posadas, Misiones Argentina. INTA, Buenos Aires, Argentina.

Michalzik, B., K. Kalbitz, J.H. Park, S. Solinger, and E. Matzner. 2001. Fluxes and concentrations of dissolved organic carbon and nitrogen: a synthesis for temperate forests. Biogeochemistry 52(2):173-205. 
Pose, N.N., M.C. Baeza, E.C. Zamuner, P.F. Di Gerónimo, y C. Videla. 2016. Parámetros agronómicos y ambientales de fósforo en suelos molisoles con diferentes usos en la provincia de Buenos Aires, Argentina. Acta Agronómica 65(4):375-382.

Reussi Calvo, N., H.R. Sainz Rozas, H.E. Echeverría, and A. Berardo. 2013. Contribution of anaerobically incubated nitrogen to the diagnosis of nitrogen status in spring wheat. Agron. J. 105(2):321-328.

Reussi Calvo, N., M. Calandroni, G. Studdert, F. Cabria, N. Diovisalvi, and A. Berardo. 2014. Nitrógeno incubado en anaerobiosis y materia orgánica en suelos agrícolas de Buenos Aires. En Actas XXIV Congreso Argentino de la Ciencia del Suelo y II Reunión Nacional Materia Orgánica y Sustancias Húmicas. 5 a 9 de mayo de 2014. Bahía Blanca, Buenos Aires, Argentina. [CD-Rom]. Asociación Argentina de la Ciencia del suelo y Universidad Nacional del Sur, Bahía Blanca, Buenos Aires, Argentina.

Rhoades, J.D. 1996. Salinity: Electrical conductivity and total dissolved solids. p. 417-435. In Page, A.I., R.H. Miller, and R.H. Keeney (eds.). Methods of Soil Analysis. Chemical and Microbiological Methods. American Society of Agronomy, Madison, Wisconsin, USA

Robertson, G.P., and P.M. Groffman. 2015. Nitrogen transformations. p. 421-446. In E.A. Paul (ed.). Soil microbiology, ecology and biochemistry. $4^{\text {th }}$ ed. Academic Press, Burlington, Massachusetts, USA.

Rodríguez S., C. Videla, E.C. Zamuner, L.I. Picone, N.N. Pose, y N.O. Maceira. 2015. Cambios en propiedades químicas de un suelo Molisol de la Región Pampeana argentina con diferente historia de manejo. Chil. J. Agric. Anim. Sci. 31:137-148

Sainz Rozas, H.R., H.E. Echeverria, y H.P. Angelini. 2011. Niveles de materia orgánica y pH en suelos agrícolas de la Región Pampeana y Extrapampeana. Cienc. Suelo 29(1):29-37.

Sainz Rozas, H., H.E. Echeverría, y H. Angelini. 2012. Fósforo disponible en suelos agrícolas de la región pampeana y extra pampeana argentina. Rev. Investig. Agropec. 38(1):33-39.

SAS Institute Inc. 2008. User Installation Guide for SAS® 9.1.3 Foundation for Microsoft ${ }^{\circledR}$ Windows®. Cary, North Carolina, USA.

Schlichting, E., H.P. Blum, and K. Stahr. 1995. BodenkundlichesPrakticum. 295 p. Paul Parey Verlag, Berlin, Germany

Sequeira, C.H., and M.M. Alley. 2011. Soil organic matter fractions as indices of soil quality changes. Soil Sci. Soc. Am. J. 75(5):1766-1773.
Soil Survey Staff. 2014. Keys to Soil Taxonomy. 12th ed. USDA, Natural Resources Conservation Service, Washington D.C., USA.

Summer, M.E., and W.P. Miller. 1996. Cation exchange capacity and exchange coefficients. p. 1201-1229. In Page, A.I., R.H. Miller, and R.H., Keeney (eds.). Methods of Soil Analysis. Chemical and Microbiological Methods. American Society of Agronomy, Madison, Wisconsin, USA.

Thomas, G.W. 1996. Exchangeable cations. p. 159-165. In Page, A.I., R.H. Miller, and R.H., Keeney (eds.). Methods of Soil Analysis. Part 2. Chemical and microbiological methods. American Society of Agronomy, Madison, Wisconsin, USA.

USDA. 1996. Soil survey laboratory methods manual. 693 p. Report № 42. Version 3.0. Soil Survey Investigations. United States Department of Agriculture (USDA), Washington D.C., USA.

Vázquez, M. 2005. Calcio y magnesio del suelo. Encalado y enyesado. En Echeverría, H.E. y F.O. García (eds.). Fertilidad de suelos y fertilización de cultivos. p .161-185. Ediciones INTA, Buenos Aires, Argentina.

Videla, C., A. Pazos, P.C. Trivelin, H.E. Echeverría, and G.A. Studdert. 2005. Mineralización bruta de nitrógeno en un Molisol de Balcarce bajo labranza convencional, siembra directa y pastura. Cienc. Suelo 23(2):133-144.

Waring, S.A., and J.M. Bremner. 1964. Ammonium production in soil under waterlogged condition as an index of nitrogen availability. Nature 201:951-952.

Wiesmeier, M., D.P. Dick, C. Rumpel, R.S.D. Dalmolin, A. Hilscher, and A. Knicker. 2009. Depletion of soil organic carbon and nitrogen under Pinus taeda plantations in Brazilian grasslands (Campos). Eur. J. Soil Sci. 60(3):347-359.

Wyngaard, N., H.E. Echeverría, H.R. Sainz Rozas, and G. Divito. 2012. Long-term fertilization and tillage effects on soil properties and maize yield in a Southern Pampas Argiudol. Soil Till. Res. 119:22-30.

Zalba, P., and N. Peinemann. 1987. Efecto de algunas especies forestales sobre ciertas propiedades fisicoquímicas del suelo. Cienc. Suelo 5(1):71-16. 